Copyright by the Acoustical Society of America. Rodriguez, H. M., \& Burdisso, R. A. (1998). Structural-acoustic control system design by multi-level optimization. Journal of the Acoustical Society of America, 104(2), 926-936.

\title{
Structural-acoustic control system design by multi-level optimization
}

\author{
Héctor M. Rodríguez \\ Department of General Engineering, University of Puerto Rico, Mayagüez Campus, Mayagüez, \\ Puerto Rico 00681-9044 \\ Ricardo A. Burdisso \\ Vibration and Acoustics Laboratories, Department of Mechanical Engineering, Virginia Polytechnic \\ Institute and State University, Blacksburg, Virginia 24061-0238
}

(Received 29 August 1997; accepted for publication 2 April 1998)

\begin{abstract}
A multi-level optimization approach for the design of feedforward active structural acoustic control (ASAC) systems is presented. The formulation takes advantage that both the structural response and the acoustic radiation from a controlled structure can be completely defined in the modal domain. All the physical parameters that define the control inputs and the error sensors are defined in the modal domain in terms of the unit modal control forces and the modal error sensor components, respectively. The upper level of the optimization solves for the optimum modal parameters that minimize the total radiated acoustic power. Then, these optimum modal parameters are used in a set of lower level or physical domain optimization problems to determine the physical characteristics of the actuators and sensors to be implemented. Since the response of the system is evaluated in the upper level using a modal approach, the formulation permits the implementation of numerical techniques and/or experimental data during the design process. Therefore, the proposed methodology can be used for the design of control systems for realistic structures with complex disturbances in an efficient manner. The design formulation is illustrated for the case of a simply supported plate excited by an off-resonance disturbance. (C) 1998 Acoustical Society of America.
\end{abstract} [S0001-4966(98)03707-2]

PACS numbers: 43.40.Vn [PJR]

\section{INTRODUCTION}

An approach for the attenuation of structurally radiated noise consists in modifying the vibration behavior of the structure by applying control force inputs directly to the structure. This technique is known as Active Structural Acoustic Control (ASAC) and was first introduced by Fuller. ${ }^{1}$ The combination of this technique in conjunction with new developments in specialized actuators and sensor materials have permitted the implementation of the concept of "smart" or adaptive systems where the transducers being an integral part of the structure. At present, such advanced control systems have been successfully implemented to control sound radiation from plates and cylinders; sound transmission/radiation from single and double panels; and interior noise from cylindrical structures among others. ${ }^{2}$

Although the concept of ASAC systems along with "smart" structures has been successfully tested, the design aspects of such systems is still under much investigation. A formal optimization approach in ASAC implementations was first introduced by Wang and co-workers. ${ }^{3}$ This investigation consisted in the direct optimization of the location of rectangular piezoelectric (PZT) actuators to minimize the sound radiation from a simply supported plate. The results demonstrated that optimally located actuators provided a far better sound reduction, at both on- and off-resonance excitations, than actuators whose position were chosen based in some physical consideration. More recently, other optimum design approaches for structural-acoustic control systems have been proposed such as using subset selection techniques, ${ }^{4}$ cluster analyses, ${ }^{5}$ eigenproperty assignments, ${ }^{6}$ and genetic algorithms. ${ }^{7}$ These previous works have demonstrated that optimally designed actuators and sensors can have a significant impact on the performance of active control systems to reduce both sound radiation and acoustic fields inside enclosures. They have shown that significant levels of attenuation can be obtained with far fewer optimally located transducers, thus reducing the dimensionality and complexity of the control system. However, there are still limitations that prevent the same approaches from being implemented in the design of complex realistic structures.

For the case of minimizing sound radiation from complex structures, the design approach of ASAC systems is virtually nonexistent. In most of the previous optimization approaches, the design formulation consisted of using a nonlinear optimization algorithm to minimize an expression related to the total radiated acoustic power as a function of the physical characteristics (i.e., location, size, etc.) of the actuators and sensors. The main problem with this straightforward approach is that the computation of the acoustic response for complex structural systems as a function of the physical characteristics of the actuators and sensors results in a computationally expensive procedure.

In this work an efficient formulation for the design of feedforward ASAC systems to suppress acoustic radiation from complex structural systems under light fluid loading (i.e., no acoustic feedback) is presented. The approach consists in a two-stage multi-level optimization scheme. The for- 
mulation takes advantage of the fact that both the structural response and the acoustic radiation from a controlled structure can be completely defined in terms of modal quantities. All the physical parameters that define the control inputs and the error sensors have their counterpart in the modal domain through the unit modal control forces and the modal error sensor components, respectively. Therefore, the upper level of the optimization is defined in the modal domain and solves for the optimum unit modal control forces and modal error sensor components that minimize the total radiated power. Then, these optimum modal parameters are used in a set of lower level optimization problems to determine the physical characteristics of the actuators and sensors to be implemented. Since in the formulation the response is evaluated in the upper level or modal domain, it permits the implementation of numerical techniques and/or experimental data during the design process. In addition, since the upper level optimization problem is universal for any type of structure/transducer combination, a previously developed general analytical sensitivity formulation ${ }^{8}$ is implemented to improve the performance of the optimization algorithm during the solution process. Therefore, the formulation is capable to handle complex structures with complex disturbances (e.g., multiple frequencies) in an efficient manner. Once the optimum modal parameters are obtained, different types of transducers can be investigated with a minimum computational effort. The proposed approach is illustrated for the case of a simply supported plate excited by an offresonance disturbance. The study of a plate will serve as a benchmark to implement the design methodology for more complex systems.

\section{STRUCTURAL-ACOUSTIC RESPONSE}

\section{A. Structural response}

The response of a structure can be obtained by modal superposition once the eigenproperties of the system (i.e., natural frequencies and mode shapes) are known. These eigenproperties can be estimated using numerical techniques such as the finite element method (FEM) or experimentally using modal analysis techniques. For the case of sound radiation from structures submerged in light fluids such as air, it is necessary to consider only the vibration in which the response normal to the radiating surface is dominant. ${ }^{9}$ The Fourier transform (FT) of the response in the direction normal to the structural surface can be obtained as a linear combination of the modes as

$$
\begin{aligned}
w\left(\mathbf{r}_{s}, \omega\right) & =\sum_{n=1}^{N} q_{n}(\omega) \phi_{n}\left(\mathbf{r}_{s}\right) \\
& =\sum_{n=1}^{N} F(\omega) f_{n} H_{n}(\omega) \phi_{n}\left(\mathbf{r}_{s}\right),
\end{aligned}
$$

where $\omega$ is the excitation frequency, $\phi_{n}\left(\mathbf{r}_{s}\right)$ is the $n$th mode shape, $N$ is the total number of modes included in the analysis, $\quad \mathbf{r}_{s}$ is a point on the structure, and $q_{n}(\omega)$ $=F(\omega) f_{n} H_{n}(\omega)$ is the FT of the $n$th modal displacement where $F(\omega)$ is the amplitude of the external excitation and $f_{n}$ is the $n$th unit modal force defined as

$$
f_{n}=\int_{S} \phi_{n}\left(\mathbf{r}_{s}\right) f\left(\mathbf{r}_{s}\right) d S,
$$

where the integral is extended over the structural domain $S$ and $f\left(\mathbf{r}_{s}\right)$ is a function that defines the spatial distribution of the excitation. Finally, $H_{n}(\omega)$ is the $n$th modal frequency response function defined as $H_{n}(\omega)=\left(\omega_{n}^{2}-\omega^{2}\right.$ $\left.+2 j \beta_{n} \omega_{n} \omega\right)^{-1}$, where $j$ is the imaginary number and $\beta_{n}$ is the $n$th modal damping ratio.

\section{B. Acoustic response}

The acoustic radiation $p(\mathbf{r}, \omega)$ from a harmonically vibrating structure can be obtained after solving the Helmholtz wave equation ${ }^{10}$

$$
\nabla^{2} p(\mathbf{r}, \omega)+k^{2} p(\mathbf{r}, \omega)=0
$$

in conjunction with the boundary conditions

$$
\boldsymbol{\nabla} p\left(\mathbf{r}_{s}, \omega\right)=\omega^{2} \rho w\left(\mathbf{r}_{s}, \omega\right)
$$

and

$$
\lim _{|\mathbf{r}| \rightarrow \infty} p(\mathbf{r}, \omega) \rightarrow 0,
$$

where $\mathbf{r}$ is a field point location; $\boldsymbol{\nabla}($.$) is the Laplacian opera-$ tor; $k=\omega / c$ is the acoustic wave number; and $c$ and $\rho$ are the phase speed and density in the fluid that surrounds the structure, respectively. Equation (4a) relates the structural response with the acoustic pressure at the structural surface while Eq. (4b) represents the Sommerfeld far-field condition that requires that the acoustic pressure decreases as the field point is moved away from the structure. Equations (3) and (4) do not possess closed-form solution for any but very simple cases. For the case of structures with complex geometries, they are solved using numerical techniques such as the boundary element method (BEM). ${ }^{11}$

The strategy in this work is to obtain the acoustic response by considering the contributions of each of the structural modes separately. By substituting the modal expansion in Eq. (1) into Eq. (4), the acoustic pressure field can be obtained as a linear contribution of the structural modes as

$$
p(\mathbf{r}, \omega)=\sum_{n=1}^{N} q_{n}(\omega) p_{n}(\mathbf{r}, \omega),
$$

where $p_{n}(\mathbf{r}, \omega)$ is the acoustic pressure at $\mathbf{r}$ due to the $n$th mode shape, i.e., modal pressure.

The total acoustic radiated power can be estimated by integrating the far-field acoustic intensity over a sphere of surface area $A$ that surrounds the structure as

$$
\Pi(\omega)=\int_{A} \frac{|p(\mathbf{r}, w)|^{2}}{2 \rho c} d A .
$$

Replacing Eq. (5) into Eq. (6), the acoustic power can be written in a general quadratic form as 


$$
\begin{aligned}
\Pi(\omega) & =\sum_{n=1}^{N} \sum_{m=1}^{N} q_{n}(\omega) q_{m}^{*}(\omega) \int_{A} \frac{p_{n}(r, \omega) p_{m}^{*}(r, \omega)}{2 \rho c} d A \\
& =\{q(\omega)\}^{H}[K(\omega)]\{q(\omega)\},
\end{aligned}
$$

where the superscripts $(*)$ and $H$ imply complex conjugate and conjugate transpose, respectively; and the elements of matrix $[K(\omega)]$ are given by the integral in Eq. (7).

The diagonal elements of the matrix $[K(\omega)]$ represent the power radiated due to the direct contributions of the modes, while the off-diagonal terms contain the power radiated due to the coupling of the modes. In particular, the off-diagonal terms could be either positive or negative depending if the interaction between the modes is constructive or destructive. For the case of complex structures, this matrix has to be evaluated by numerical techniques and therefore represents the highest computational effort during the evaluation of the acoustic response. Finally, $\{q(\omega)\}$ is the vector of modal displacements.

Equation (7) defines the total radiated power at a single frequency. The general case of multiple frequency excitations is expressed as a linear combination of the contributions due to each frequency as

$$
\Pi_{T}(\omega)=\sum_{i=1}^{N_{F}}\left(\left\{q\left(\omega_{i}\right)\right\}^{H}\left[K\left(\omega_{i}\right)\right]\left\{q\left(\omega_{i}\right)\right\}\right)=\sum_{i=1}^{N_{F}} \Pi\left(\omega_{i}\right),
$$

where $N_{F}$ is the total number of frequencies.

Inspection of Eqs. (7) and (8) show that the relative contributions to the acoustic power due to the modes depend on two factors: the complex amplitudes of the modal displacements $\left\{q\left(\omega_{i}\right)\right\}$ and the relative values of the modal power matrices $\left[K\left(\omega_{i}\right)\right]$. The modal displacements $\left\{q\left(\omega_{i}\right)\right\}$ depend on the dynamics of the structure under a particular disturbance $F(\omega)$. In the same way, the matrices $\left[K\left(\omega_{i}\right)\right]$ depend on the capacity of the structural modes to generate sound and their coupling characteristics. For a mode excited on-resonance, its modal displacement will be significantly larger than the other ones and therefore this mode will most likely dominate the acoustic field. On the other hand, at offresonance conditions the acoustic field will probably be mainly due to the radiation of the modes with the higher radiation efficiency.

\section{Optimum feedforward control inputs}

Equation (1) represents the steady-state or frequency domain structural response due to an external disturbance $F(\omega)$. The response due to this disturbance can be controlled by applying $N_{c}$ secondary control forces $U_{k}(\omega)$ where $k$ $=1, \ldots, N_{c}$. The response of the controlled system can be expressed as ${ }^{6}$

$$
w^{c}\left(\mathbf{r}_{s}, \omega\right)=\sum_{n=1}^{N} q_{n}^{c}(\omega) \phi_{n}\left(\mathbf{r}_{s}\right),
$$

where, using the principle of superposition, the $n$th modal displacement of the controlled system is

$$
q_{n}^{c}(\omega)=q_{n}(\omega)+H_{n}(\omega) \sum_{k=1}^{N_{c}} U_{k}(\omega) u_{n k},
$$

where $U_{k}(\omega)$ and $u_{n k}$ are the amplitude and the $n$th unit modal control force of the $k$ th control input, respectively. As can be seen in Eq. (10), the unit modal control forces $u_{n k}$ dictate the relative controllability of the different modes by the control inputs $U_{k}(\omega)$. Similarly to the unit modal disturbances, the unit modal control forces are given as

$$
u_{n k}=\int_{S} \phi_{n}\left(\mathbf{r}_{s}\right) u_{k}\left(\mathbf{r}_{s}\right) d S,
$$

where $u_{k}\left(\mathbf{r}_{s}\right)$ defines the spatial distribution of the control forces. Similar to the unit modal disturbances, the unit modal control forces depend completely on the physical implementation of the actuators, e.g., shakers, PZT ceramics, etc.

In feedforward control, the complex amplitude of the control inputs $U_{k}(\omega)$ are obtained by "feeding forward" a reference signal $x(\omega)$, fully coherent to the original disturbance $F(\omega)$, through an array of compensators. The compensators are designed such that a measurable control cost function is minimized. This cost function is usually obtained as the sum of the mean-square-value (msv) of the response at the error sensors as

$$
J=\sum_{s=1}^{N_{s}}\left|E_{s}(\omega)\right|^{2},
$$

where $E_{s}(\omega)$ is the FT of the response due to the $s$ th error sensor output and $N_{s}$ is the total number of error outputs.

In ASAC applications, the error signals could be obtained by measuring directly the acoustic field using microphones or the structural vibration using structural sensors. In many applications the use of far-field microphones as error sensors is not practical. Since one of the objectives in this work is to eliminate the need for the use of microphones and design a completely adaptive system with the actuators and sensors integrated to the structure, only structural sensors are considered here. For the case of such sensors, the response can always be expressed as a linear combination of modal terms as ${ }^{6}$

$$
E_{s}(\omega)=\sum_{n=1}^{N} q_{n}^{c}(\omega) \xi_{n s}
$$

where $\xi_{n s}$ is the $n$th modal component of the $s$ th error sensor. Again, the modal error components are related to the physical implementation of the sensors and they dictate the relative observability of the different modes

By differentiating Eq. (13) with respect to the real and imaginary part of the control inputs $U_{k}(\omega)$ and setting them to zero, it can be shown that the set of the optimum control inputs is the solution of the following linear system of equations

$$
\left[T_{c e}(\omega)\right]\{U(\omega)\}=-F(\omega)\left\{T_{d e}(\omega)\right\},
$$

where vector $\left\{U_{k}(\omega)\right\}$ contains the $N_{c}$ control inputs, the elements of matrix $\left[T_{c e}(\omega)\right]$ are the transfer functions be- 
tween the control inputs and the error outputs and the elements of vector $\left\{T_{d e}(\omega)\right\}$ contain the transfer functions between the disturbances and the error outputs.

Once the optimum feedforward control inputs $U_{k}(\omega)$ are obtained from the solution of Eq. (14) they are substituted into Eq. (10) to compute the modal displacements of the controlled system $q_{n}^{c}(\omega)$. Finally, the controlled modal displacements can be substituted for the uncontrolled modal displacements in Eq. (7) to yield the total radiated power of the controlled system as

$$
\Pi^{c}(\omega)=\left\{q^{c}(\omega)\right\}^{H}[K(\omega)]\left\{q^{c}(\omega)\right\},
$$

where $\left\{q^{c}(\omega)\right\}$ is the vector of controlled modal displacements. In the same way, the total controlled radiated power due to the contribution of $N_{F}$ frequencies is given as

$$
\begin{aligned}
\Pi_{T}^{c}(\omega) & =\sum_{i=1}^{N_{F}}\left(\left\{q^{c}\left(\omega_{i}\right)\right\}^{H}\left[K\left(\omega_{i}\right)\right]\left\{q^{c}\left(\omega_{i}\right)\right\}\right) \\
& =\sum_{i=1}^{N_{F}} \Pi^{c}\left(\omega_{i}\right) .
\end{aligned}
$$

Equation (16) describes the acoustic response of a feedforward controlled structure. This equation clearly shows the advantage of evaluating the response as a combination of modal contributions, especially in terms of the computational effort. This advantage is specially critical during the design of the control system. This design procedure involves a systematic update of the physical characteristics of the transducers (i.e., type, size, number, location, etc.) and the evaluation of the acoustic response for each configuration. This process is continued until the desired attenuation is obtained. Using Eq. (16), the evaluation of the acoustic response is just a matter of simple algebraic manipulations once the elements of matrices $\left[K\left(\omega_{i}\right)\right]$ are known.

\section{CONTROL SYSTEM DESIGN BY MULTI-LEVEL OPTIMIZATION}

As shown in Eq. (16), the total radiated power from the controlled system is a function of the forces $U_{k}(\omega)$ which in turn are defined from the modal characteristics of the uncontrolled system, i.e., $F(\omega) f_{n}$ and $H_{n}(\omega)$, and of the actuators and sensors, i.e., $u_{n k}$ and $\xi_{n s}$. The unit modal control forces $u_{n k}$ and modal error sensor components $\xi_{n s}$ are directly related to the physical implementation of the actuators and sensors, respectively. Therefore, the design of the control system consists in finding the transducers that, when implemented, yield the proper unit modal control forces $u_{n k}$ and modal error sensor components $\xi_{n s}$. The significance of the unit modal control forces and the modal error sensor components in the response of the controlled system is that they represent the relative controllability and observability of the modes by the control actuators and the error sensors, respectively. Regardless of the type of actuators and sensors, the unit modal control forces and the modal error sensor components play the role of weighting coefficients that can be normalized between \pm 1 .
The fact that the response of the controlled system can be obtained without the knowledge of the physical characteristics of the transducers but from their effect on the different modes brings out the opportunity of designing the control system using a multi-level optimization approach. In multilevel optimization a complex problem is broken into a set of simpler problems that offers computational advantages. The process of decomposition consists of separating the optimization process in an upper level problem, in which a global cost function is minimized with respect to global design variables, and a set of lower level problems, in which a set of local design variables are related to all the global design variables or a subset of them. The upper level or modal domain optimization consists in finding the optimum unit modal control forces and modal error sensor components that minimize the total radiated acoustic power. Therefore, at the upper level optimization, the global cost function is the total radiated acoustic power as presented in Eq. (16) and the global design variables are the modal quantities $u_{n k}$ and $\xi_{n s}$. Once the optimum modal parameters are obtained, a set of lower level or physical domain optimization problems can be solved in which the actuators and sensors that yield the optimum unit modal control forces $u_{n k}$ and modal error sensor components $\xi_{n s}$ are obtained. Therefore, the local design variables at the lower level are the physical parameters that define the actuators and sensors, i.e., number, size, location, etc., while the local cost functions are the sum of the squares of the differences between the optimum modal quantities and the "actual" modal parameters due to the implementation of a particular transducer. The outcome of this procedure is an optimally designed control system.

The main advantages of the proposed design approach can be listed as:

(1) The design process is broken into the solution of two simple problems. The modal domain or upper optimization problem consists in the minimization of a continuous function of the modal control parameters. On the other hand, the physical domain or lower level formulations consist in a set of much simpler problems that involves matching the "actual" modal parameters of the transducers as closely as possible to the "ideal" modal parameters. The simplicity in the solution in this twolevel approach should be contrasted to the difficulty in solving a straightforward optimization problem in which the acoustic response is directly expressed in terms of the physical characteristics of the transducers.

(2) Since the upper level is solved in the modal domain, the formulation is applicable to the design of any complex structure modeled using FEM/BEM codes or using experimental modal analysis data. The highest computational effort at this level is in computing the matrices $\left[K\left(\omega_{i}\right)\right]$ using Eq. (7). The computation of these matrices has to be carried out only once for each disturbance frequency during the design process.

(3) Since the modal domain is universal for any type of structure, a sensitivity analysis of the cost function and the constraints can be developed in this stage. The use of 
analytical sensitivities instead of numerical approximations greatly improves the performance of optimization algorithms. ${ }^{8}$

(4) Since the response of the controlled system is completely defined in the modal domain, the upper level formulation can be used to investigate and evaluate the performance of different control system configurations. At this level, the designer can decide with relative ease the required number of control inputs $N_{c}$ and error outputs $N_{s}$ that are necessary to obtain a desired attenuation.

(5) Since the design of the transducers to be implemented in the control system is carried out after solving simple sets of optimization problems, different models of actuators and sensors can be easily investigated at the lower level without the need to recompute the response of the structure.

\section{A. Upper level or modal domain optimization}

The modal domain or upper level optimization problem is posed as follows:

$$
\operatorname{Min} R\left(u_{n k}, \xi_{n s}\right)=\frac{\Pi_{T}^{c}(\omega)}{\Pi_{T}(\omega)}
$$

such that

$$
\begin{gathered}
g_{m}^{c}\left(u_{n k}, \xi_{n s}\right)=\sum_{n=1}^{N} \frac{\left|U_{k}\left(u_{n k}, \xi_{n s}, \omega_{i}\right) \cdot u_{n k}\right|^{2}}{\left|F\left(\omega_{i}\right) \cdot f_{n}\right|^{2}} \leqslant \lambda_{m}, \\
i=1, \ldots, N_{F} \quad m=N_{c} \times N_{F} \\
g_{k}^{n}\left(u_{n k}\right)=\sum_{n=1}^{N} u_{n k}^{2}=1, \quad k=1, \ldots, N_{c} \\
g_{s}^{\xi}\left(\xi_{n s}\right)=\sum_{n=1}^{N} \xi_{n s}^{2}=1, \quad s=1, \ldots, N_{s} .
\end{gathered}
$$

The optimization problem stated in Eq. (17) seeks to minimize the ratio $R\left(u_{n k}, \xi_{n s}\right)$ of the total radiated power of the controlled system $\Pi_{T}^{c}(\omega)$ to the total radiated power of the uncontrolled system $\Pi_{T}(\omega)$. The inequality constraints in Eq. (17b) impose restrictions on the effort, through the penalty parameter $\lambda_{m}$. By setting the penalty parameter $\lambda_{m}$ to less than one, Eq. (17b) implies that the modulus square of each one of the modal control forces, $\left|U_{k}\left(\omega_{i}\right) u_{n k}\right|^{2}$, will be in a weighted average sense less than the modulus square of the modal disturbances, $\left|F\left(\omega_{i}\right) f_{n}\right|^{2}$. Therefore, by satisfying these constraints the control force applied to each mode will be less than the modal force due to the disturbance. There is an inequality constraint of this type for each one of the $N_{c}$ control inputs at each one of the $N_{F}$ frequencies. It is important to mention that the constraints in the control effort Eq. (17b) could also be implemented as a penalty in the cost function. Finally, the equality constraints in Eqs. (17c), (17d) imply a normalization of the design variables since the only relevant information is their relative values, which defines the relative controllability and observability of the modes, respectively. This continuous optimization problem in the upper level or modal domain can be solved by any con- strained optimization algorithm in conjunction with previously developed analytical sensitivity formulations. ${ }^{8}$

\section{B. Lower level or physical domain optimization}

Once the optimum modal parameters are obtained they are used as goals to reach in a set of physical domain optimization problems. The purpose of the optimization problems at this level is to find the physical characteristics of the desired actuators and sensors that when implemented in the real structure induce unit modal control forces and modal error sensor components as close as possible to the optimum values obtained from the upper level optimization. For the sake of clarity, from now on the optimum modal parameters obtained in the upper level are denoted as "ideal" while the modal parameters of the physical transducers are denoted as "actual." The "actual" modal parameters are functions of the physical design parameters of the transducers such as type, location, dimensions, etc. Hence, the first requirement at this level is to have mechanical models for the transducers that relate the modal parameters to the physical design variables. In addition, a single control input (error output) can be implemented using multiple actuators (sensors) wired in- or out-of-phase. Therefore, the cost functions at this level are a measure of the difference between the "ideal" and "actual" modal parameters while the design variables are the physical characteristics of the implementation of the transducers (i.e., size, location, relative phases, etc.).

\section{Optimum actuator design}

The set of lower level or physical domain optimization problems to obtain the optimum physical design parameters $\{a\}$ for the control actuators can be expressed in formal optimization notation as

$$
\min C_{k}(\{a\})=\sum_{n=1}^{N}\left|u_{n k}-\hat{\Psi}_{n k}(\{a\})\right|^{2},
$$

where the cost function $C_{k}(\{a\})$ represents a measure of the error between the "ideal" optimum unit modal control forces $u_{n k}$ and the "actual" unit modal control forces given by the function $\hat{\Psi}_{n k}(\{a\})$ for the $k$ th control input. Then, the $n$th component of the "actual" unit modal control force $\hat{\Psi}_{n k}(\{a\})$ driven by the $k$ th control signal is obtained by including the contributions of all the $n$th unit modal forces produced by each one of the $N_{A}$ actuators as

$$
\hat{\Psi}_{n k}(\{a\})=\sum_{i=1}^{N_{A}} \Psi_{n i}\left(\{a\}_{i}\right),
$$

where $\Psi_{n i}\left(\{a\}_{i}\right)$ is the mechanical model that defines the $n$th unit modal force produced by the $i$ th actuator and vector $\{a\}_{i}$ contains the design variables for the $i$ th actuator.

\section{Optimum sensor design}

Similarly, the set of lower level or physical domain optimization problems to obtain the optimum physical design parameters for the error sensors can be expressed as 
TABLE I. Modal properties of simply supported plate and modal control components.

\begin{tabular}{|c|c|c|c|c|c|c|}
\hline $\begin{array}{c}\text { Mode } \\
\left(n_{x}, n_{y}\right)\end{array}$ & $\begin{array}{c}\text { Nat. freq. } \\
\mathrm{Hz}\end{array}$ & $f_{n}$ & $\begin{array}{c}\text { "Ideal" } \\
u_{n l}\end{array}$ & $\begin{array}{c}\text { "Ideal" } \\
\xi_{n l}\end{array}$ & $\begin{array}{c}\text { "Actual" } \\
u_{n l}\end{array}$ & $\begin{array}{c}\text { "Actual" } \\
\xi_{n l}\end{array}$ \\
\hline$(1,1)$ & 85.9 & 1.370 & -0.070 & 0.161 & 0.144 & 0.380 \\
\hline$(2,1)$ & 184.9 & -1.100 & -0.166 & -0.235 & -0.290 & -0.069 \\
\hline$(1,2)$ & 244.8 & 0.570 & 0.005 & 0.359 & -0.022 & -0.146 \\
\hline$(2,2)$ & 343.8 & -0.460 & -0.002 & -0.102 & 0.050 & -0.274 \\
\hline$(3,1)$ & 349.9 & -0.480 & -0.002 & 0.186 & -0.048 & 0.096 \\
\hline$(3,2)$ & 508.5 & -0.200 & 0.001 & 0.094 & -0.053 & -0.295 \\
\hline$(1,3)$ & 509.5 & -1.130 & -0.154 & -0.143 & -0.122 & -0.124 \\
\hline$(4,1)$ & 581.0 & 1.490 & 0.963 & -0.460 & 0.927 & 0.451 \\
\hline$(2,3)$ & 608.5 & 0.910 & 0.127 & 0.455 & 0.130 & 0.500 \\
\hline$(4,2)$ & 739.8 & 0.620 & 0.002 & 0.534 & -0.036 & -0.218 \\
\hline$(3,3)$ & 773.5 & 0.400 & 0.001 & -0.110 & 0.030 & 0.380 \\
\hline
\end{tabular}

$$
\min C_{s}(\{b\})=\sum_{n=1}^{N}\left|\xi_{n s}-\hat{\Omega}_{n s}(\{b\})\right|^{2},
$$

where $C_{s}$ is the cost function relating the difference between the optimum modal error sensor components $\xi_{n s}$ and the “actual" modal error sensor components given by $\hat{\Omega}_{n s}(\{b\})$ connected to the $s$ th error channel. Similar to the case of the unit modal control forces, the $n$th component of the "actual" modal error sensor component due to the $s$ th error signal $\hat{\Omega}_{n s}(\{b\})$ can be obtained by including the contributions of all the $n$th modal error sensor components due to each one of the $N_{E}$ error sensors as

$$
\hat{\Omega}_{n s}(\{b\})=\sum_{j=1}^{N_{E}} \Omega_{n j}\left(\{b\}_{j}\right),
$$

where $\Omega_{n j}\left(\{b\}_{j}\right)$ is the $n$th modal error sensor component of the $j$ th error sensor and vector $\{b\}_{j}$ contains the design variables for the $j$ th sensor.

Equations (18) and (21), solved for each one of the $N_{c}$ control and $N_{s}$ error channels, define completely the set of lower level or physical domain optimization problems. As can be seen, once the optimum modal parameters are obtained in the upper level optimization different actuators and sensor models can be investigated with a minimum computational effort.

\section{NUMERICAL RESULTS}

This section illustrates the implementation of the proposed multi-level optimization design approach for the design of an optimum control system for a baffled simply supported plate. Since both the structural and acoustic responses of a simply supported plate can be obtained analytically, the study of this structure is ideal as a benchmark for more complex systems. The plate is made of steel with a modulus of elasticity $E=2 \times 10^{11} \mathrm{~N} / \mathrm{m}^{2}$, mass density $\rho_{s}=7800$ $\mathrm{Ns}^{2} / \mathrm{m}^{4}$, and Poisson's ratio $\nu=0.28$. The dimensions of the plate are $L_{x}=0.38 \mathrm{~m}, L_{y}=0.30 \mathrm{~m}$, and thickness $h=0.002$ $\mathrm{m}$. The plate is assumed to be vibrating in air with a density $\rho=1.21 \mathrm{~kg} / \mathrm{m}^{3}$ and phase speed $c=343 \mathrm{~m} / \mathrm{s}$. The disturbance is assumed to be a point force with amplitude $F(\omega)=1 \mathrm{~N}$ and frequency $\omega=3454 \mathrm{rad} / \mathrm{s}\left(\right.$ i.e., $550 \mathrm{~Hz}$ ) and located at $x_{f}$
$=0.24 \mathrm{~m}, y_{f}=0.13 \mathrm{~m}$. The case of multiple frequency excitations has already been studied ${ }^{12}$ and will be reported in a future publication.

\section{A. Uncontrolled system response}

For the case of a simply supported plate, the natural frequency and mass normalized mode shape, identified by their modal indices $\left(n_{x}, n_{y}\right)$, are easily computed. ${ }^{10}$ In the present case, it is assumed a modal damping ratio of $1 \%$ (i.e., $\beta_{n}=0.01$ ) for all the modes. A total of 11 modes (i.e., $N$ $=11)$ are considered in the analysis. The natural frequencies and unit modal disturbance force, $f_{n}$, are summarized in the second and third column in Table I. From the values of the natural frequencies, it can be seen that the disturbance excitation is off-resonance between the $(1,3)$ and the $(4,1)$ modes. In addition, the values of the unit modal disturbances indicate that the disturbance couples well with modes $(4,1)$, $(1,1),(1,3)$, and $(1,2)$ while is less effective in driving, for example, modes $(3,2)$ and $(3,3)$.

Similarly, the far-field acoustic response due to the $n$th mode of a baffled simply supported plate can also be obtained analytically from the solution of the Raleigh integral as $^{9}$

$$
\begin{aligned}
p_{n}(r, \theta, \gamma, \omega) & \\
= & -\omega^{2} \rho \frac{L_{x} L_{y}}{2 \pi r n_{x} n_{y}} \sqrt{\frac{4}{\rho_{s} h L_{x} L_{y}}}\left(\frac{(-1)^{n_{x}} e^{-j \tau_{1}}-1}{\left(\tau_{1} / n_{x} \pi\right)^{2}-1}\right) \\
& \times\left(\frac{(-1)^{n_{y}} e^{-j \tau_{2}}-1}{\left(\tau_{2} / n_{y} \pi\right)^{2}-1}\right) e^{-j k r},
\end{aligned}
$$

where $\quad k=\omega / c ; \quad \tau_{1}=k L_{x} \sin \theta \cos \gamma \quad$ and $\quad \tau_{2}=k L_{y}$ $\times \sin \theta \sin \gamma$; and $\theta$ and $\gamma$ are the angles defining the far-field direction.

Using Eq. (22), the contribution due to the modes, both direct and cross terms, to the total radiated power at $550 \mathrm{~Hz}$ including the dynamics of the system can be presented graphically. To this end, each one of the elements in the double summation in Eq. (7) is shown in Fig. 1. The results are presented in decibels $\left(\mathrm{dB}\right.$ ref $\left.1 \times 10^{-12} \mathrm{~W}\right)$ where the negative contributions (i.e., light shaded columns) are included in the figure as the $\mathrm{dB}$ of the absolute value. This figure shows that mode $(4,1)$ is the highest contributor to the 


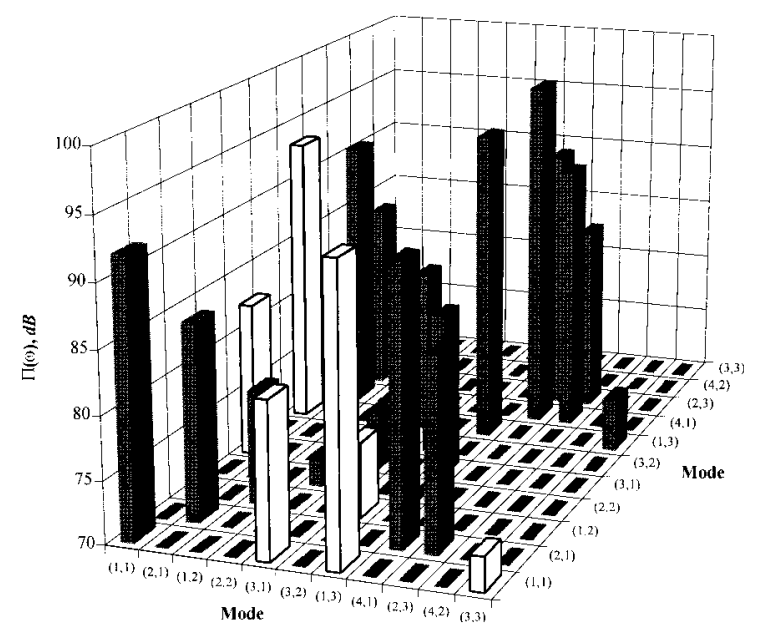

FIG. 1. Modal contributions to total radiated power. Total radiated power: $101.8 \mathrm{~dB}$.

total radiated power in spite of having low radiation efficiency. This is due to the fact that the force strongly drives this mode. These results demonstrate the significant effect that the disturbance plays in the modal contributions to the total radiated power, in particular for spectrally white inputs. On the other hand, the effect of the radiation efficiency can be observed in the fact that even though the excitation frequency is far away from the resonance of the $(1,1)$ mode the power due to this mode is only about $6 \mathrm{~dB}$ below the power produced by mode $(4,1)$. Thus this shows that both the dynamics and the radiation properties of the modes are important in the modal breakdown of the total acoustic power.

\section{B. Control system design: Results and discussion}

\section{Modal domain design}

The goal in the upper level or modal domain optimization is to find the optimum values of the unit modal control forces and modal error sensor components that minimize the total radiated power of the controlled system $\Pi_{T}^{c}(\omega)$ in the presence of designer selected constraints in the modal control effort. In the present example, this optimization problem has been solved using the Goal Attainment Method. ${ }^{13}$ Six different control configurations were studied: $1 \mathrm{I} 1 \mathrm{O}, 1 \mathrm{I} 2 \mathrm{O}$, $1 \mathrm{I} 3 \mathrm{O}, 2 \mathrm{I} 2 \mathrm{O}, 2 \mathrm{I} 3 \mathrm{O}$, and $3 \mathrm{I} 3 \mathrm{O}$ where " $\mathrm{I}$ " and " $\mathrm{O}$ " denote control and error channels, respectively. The acoustic power reduction for each of the six control configurations as a function of the penalty in the control effort are shown in Fig. 2. Each one of the configurations in the figure represents a set of optimum unit modal control forces $u_{n k}$ and optimum modal error sensor components $\xi_{n s}$. It can be observed that for a particular number of control channels, there is no increase in the reduction in the total radiated power by increasing the number of error outputs. For that reason, the curves for configurations with identical number of control inputs are overlapped in the figure (e.g., curves 1I1O, 1I2O, and 1I3O). As can be seen in the figure, the reduction in the total radiated power can be accomplished by relaxing the constraint in the control effort and/or increasing the number of control channels. The results in Fig. 2 are very useful during the

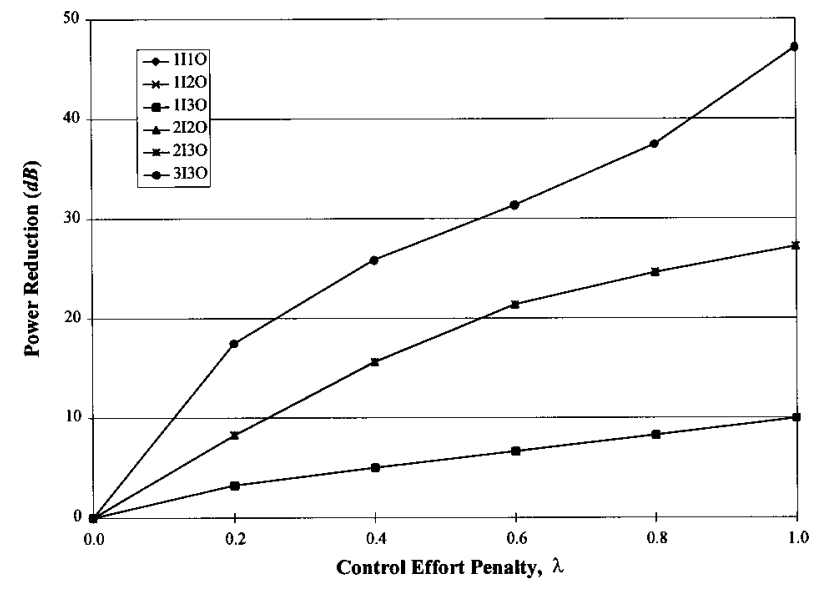

FIG. 2. Reductions in total radiated power.

design process since the designer can decide at a very early stage how many control channels are needed and what relative expense (modal control effort) is required to accomplish a desired attenuation. Since the acoustic response (before and after control) is expressed in terms of modal parameters, the same reductions will be expected for any type of actuator/ sensor configuration that yields the same unit modal control forces and modal error sensor components as the optimum ones. This is one of the main advantages of the present approach versus optimizing directly the physical characteristics of the transducers.

In order to investigate in detail the optimum results, the values for the optimum modal parameters for the $1 \mathrm{I} 1 \mathrm{O}$ configuration with a $\lambda=1.0$ are also presented in the fourth and fifth columns of Table I. As shown in the table, the optimum unit modal control forces, $u_{n k}$, suggest that most of the control effort should be dedicated to mode $(4,1)$ with significantly less effort into modes $(2,1),(1,3),(2,3)$, and $(1,1)$. This behavior is explained by plotting the modal contributions to the controlled total radiated power. This is shown in Fig. 3. Comparison of this figure and Fig. 1 shows that the mechanism of control is by mainly reducing the contribution due to mode $(4,1)$. As shown in Fig. 1 , mode $(4,1)$ is the

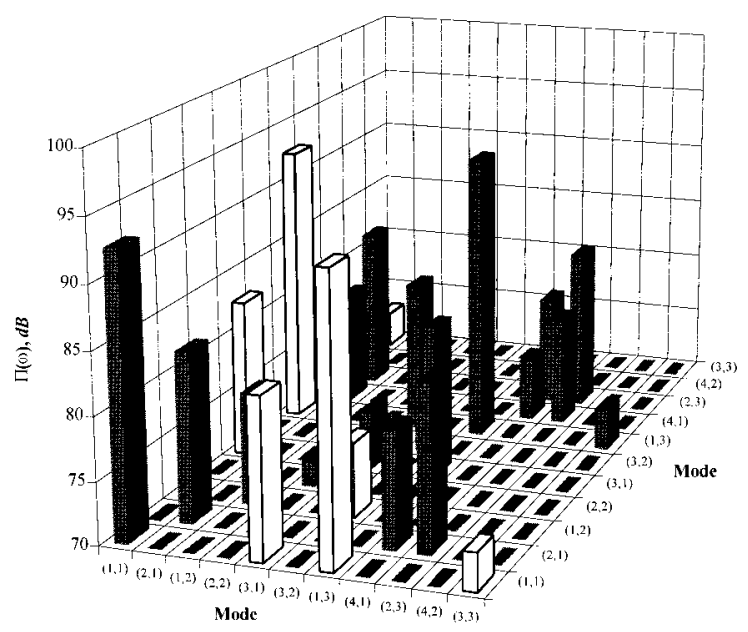

FIG. 3. Modal contributions to controlled power using the 1 IIO configuration and $\lambda=1.0$. Total radiated power: $91.8 \mathrm{~dB}$. 


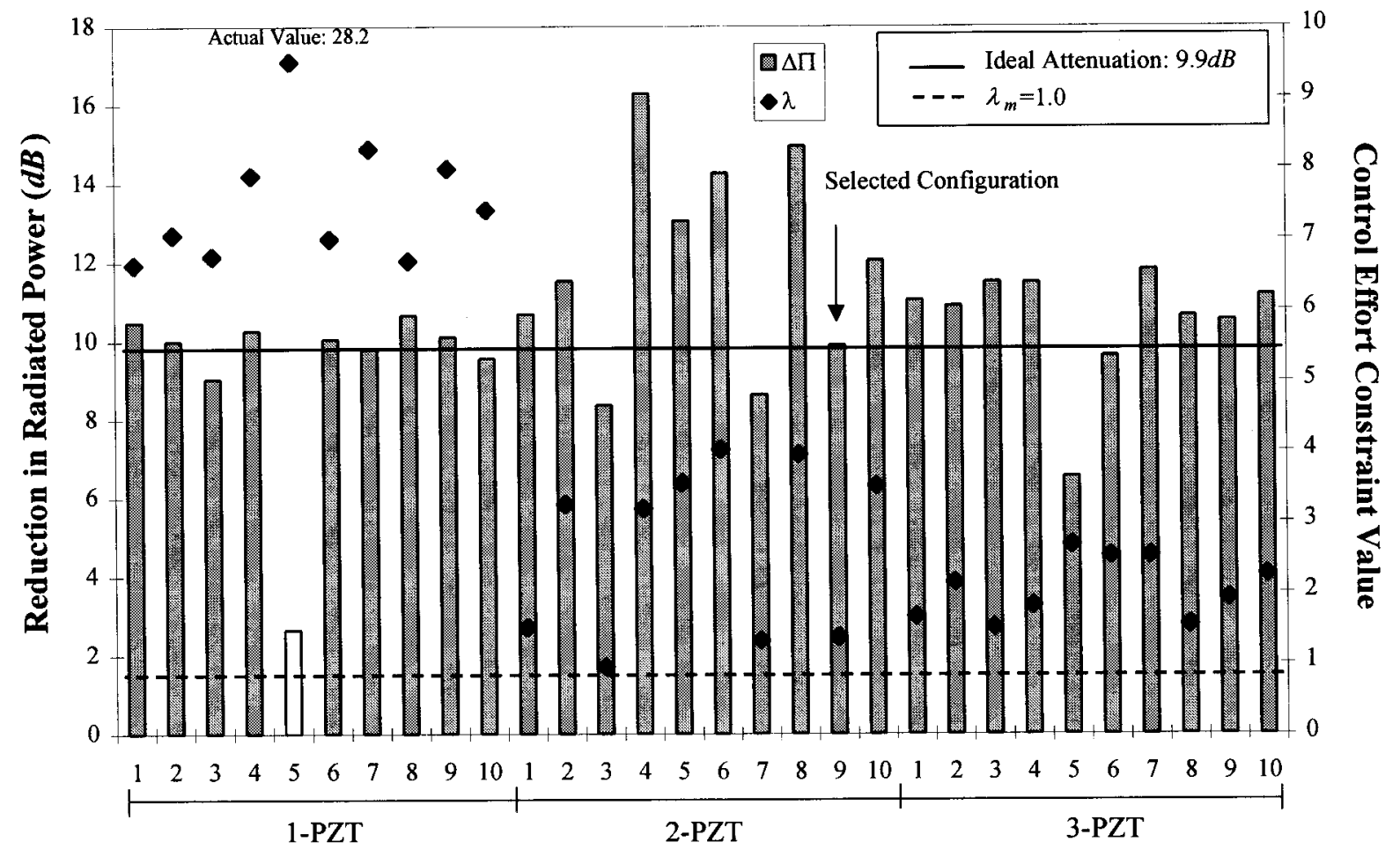

FIG. 4. Performance of optimum actuator configurations (using "ideal", sensor).

highest contributor to the uncontrolled sound field and therefore it is trivial that this mode has to be highly controlled as implied in Table I. On the other hand, modes $(1,1)$ and $(1,3)$ are virtually left unaffected by the control system as indicated by the low values of the unit modal control forces for these modes in Table I. This is because in the uncontrolled system the direct power radiated by these modes is canceled by their negative cross-radiation terms as shown in Fig. 1. On the other hand, Fig. 1 also shows that modes $(2,1)$ and $(2,3)$ do not have a significant direct term contribution to the total power. However, these modes couple acoustically with the $(4,1)$ mode as shown by the important positive cross terms in the same figure. Thus the control system attenuates the $(2,1)$ and $(2,3)$ modes to reduce these cross terms.

The previous results show that the optimization process is successful in identifying only those modes that had to be controlled to reduce the total radiated power. On the other hand, the optimum modal error sensor components in Table I do not seem to observe the more important modes in the total radiated power, where the highest value of the modal error components is for mode $(4,2)$ which is barely excited by the disturbance and do not contribute at all to the acoustic field. This is explained by looking once more at the form of Eq. (10). If the values of the control inputs $U_{k}(\omega)$ are fixed in this equation, as is the case when the modal control effort constraints are active, the only control parameters that change from mode to mode are the unit modal control forces $u_{n k}$. Therefore, the selection of the correct unit modal control forces has a direct impact on the total radiated acoustic power. On the other hand, the relevance of the optimum modal error sensor components stands in providing the cor- rect complex amplitudes for the control inputs and not in observing a particular mode due to its radiation characteristics. This also explains why there is no improvement in the reduction in the radiated power by simply increasing the number of error signals. Having more error signals will only provide different means to satisfy the required control effort constraints.

At this point, the major aspects of the modal domain optimization results have been covered. In a typical implementation of the proposed design approach, the designer investigates at the modal domain level many configurations, as shown in Fig. 2, and selects the one that best fits the particular situation (i.e., attenuation, number of control channels, control effort, etc.). Then, the optimum unit modal control forces and modal error sensor components of the selected configuration are used in the physical domain level to design the physical characteristics of the actuators and sensors.

\section{Physical domain design}

The first step in the lower level or physical domain design is the selection of the type of transducers to be used. One of the advantages of the present design approach is that different models of actuators and sensors can be investigated with a low computational effort. In the present work, the design formulation is demonstrated only for one type of actuator and one type of sensor. The control actuators will be PZT patches while the error sensors are assumed to be accelerometers. 


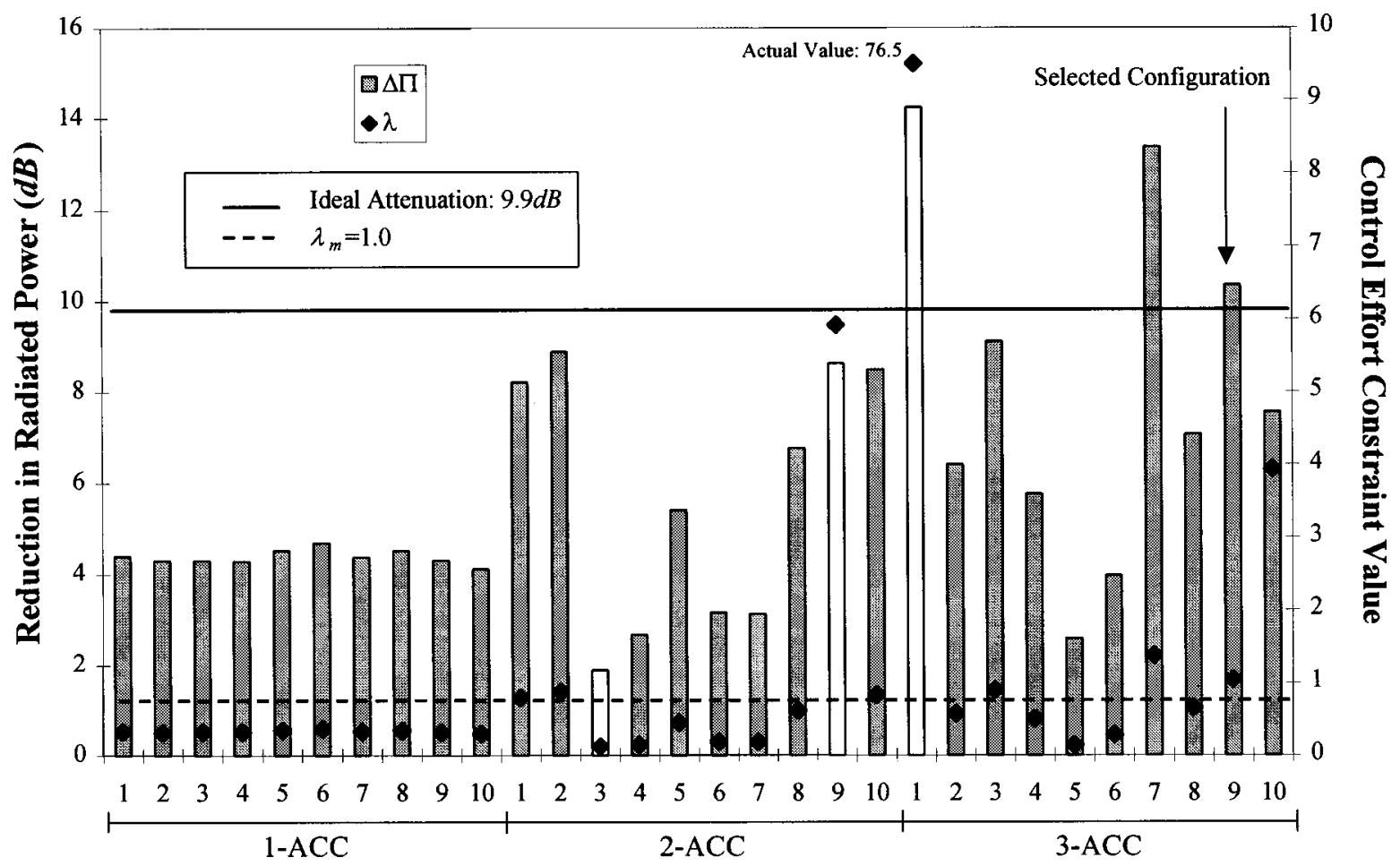

FIG. 5. Performance of optimum sensor configurations (using "ideal" control forces).

a. Optimum actuator design. A mechanical model for the PZT elements that relates the physical to the modal parameters is required. As shown in the literature, if two PZT patches are symmetrically bonded to both sides of the structure and wired out-of-phase, their effect can be approximated as line moments applied to the middle surface of the structure along the edges of the patches. ${ }^{12}$ In the present example, this configuration (i.e., a PZT pair wired out-of-phase) is denoted as a single actuator. For the case of a simply supported plate, the line moments along the edges of the $i$ th PZT actuator will yield an $n$th unit modal force in the form of ${ }^{14}$

$$
\begin{aligned}
\Psi_{n i}\left(x_{c i}, y_{c i}, l_{x i}, l_{y i}, P_{i}\right) \\
=P_{i} C_{0} \frac{k_{x n}^{2}+k_{y n}^{2}}{k_{x n} k_{y n}}\left(\cos \left(k_{x n}\left(x_{c i}-l_{x i}\right)\right)\right. \\
\left.\quad-\cos \left(k_{x n}\left(x_{c i}+l_{x i}\right)\right)\right)\left(\cos \left(k_{y n}\left(y_{c i}-l_{y i}\right)\right)\right. \\
\left.\quad-\cos \left(k_{y n}\left(y_{c i}+l_{y i}\right)\right)\right),
\end{aligned}
$$

where $P_{i}= \pm 1$ is the relative phase between actuators; the constant $C_{0}$ is a function of the thickness and material properties of the plate and the PZT elements; $k_{x n}=n_{x} \pi / L_{x}$ and $k_{y n}=n_{y} \pi / L_{y}$; and $\left(x_{c i}, y_{c i}\right)$ and $\left(l_{x i}, l_{y i}\right)$ are the coordinates of the central location and dimensions of the $i$ th PZT pair, respectively. Using this configuration for the unit modal control forces, the optimum control inputs $U_{k}(\omega)$ will be the voltages to be applied to the actuators. The properties of the PZT patches used in this example are: piezoelectric strain coefficient $d_{31}=171 \times 10^{-12} \mathrm{~m} / \mathrm{V}$, Poisson's ratio $v_{p}=0.31$, Young's modulus $E_{p}=6.1 \times 10^{10} \mathrm{~N} / \mathrm{m}^{2}$, and thickness $h_{p}$ $=0.0002 \mathrm{~m}$. Finally, Eq. (23) is used to obtain the $n$th unit modal force due to $N_{A}$ actuators driven in- or out-of-phase by the control input.
As implied in Eq. (23), the actuator design variables [i.e., the elements of $\{a\}_{i}$ in Eq. (18)] are the central location $\left(x_{c i}, y_{c i}\right)$, the dimensions $\left(l_{x i}, l_{y l}\right)$, and the relative phase $P_{i}$ for the $i$ th PZT pair. In the present example, up to three PZT pairs (i.e., $N_{A}=1,2$ or 3 ) were considered in the design formulation. A genetic algorithm ${ }^{15}$ (GA) was used to solve this problem. Ten different GA analyses were carried out for each one of the PZT configurations. The best solution from each analysis was selected that yielded a final set of thirty possible control implementations.

The performance of these thirty control configurations is summarized in Fig. 4. The "actual" modal error sensor components are not known at this stage, therefore the results shown in this figure are obtained out using the "ideal" values of the modal error components $\xi_{n s}$ given in Table I. In this figure, the horizontal axis represents the 30 control configurations, e.g., the first ten are the best solutions for each one of the GA analyses with a single actuator and so forth. The columns represent the power reduction achieved for each configuration. For comparison purposes, the solid horizontal line represents the predicted power attenuation from the modal domain optimization [i.e., $\Delta \Pi(\omega)=9.9 \mathrm{~dB}$ ]. The control effort constraint given in Eq. (17b) is also plotted (dotted line) to illustrate the performance of the actuator configurations. The limit of this constraint (i.e., $\lambda=1.0$ ) is indicated by the horizontal dotted line. Thus any value higher that 1.0 indicates that the constraint is violated.

The results in Fig. 4 show that good attenuation in the total radiated power is obtained even when using a single PZT. The fifth case gives an increase in the power (i.e., clear column implies negative reduction) but since there are many other alternate solutions, the designer just needs to discard that one. The good performance of the single PZT is at the 


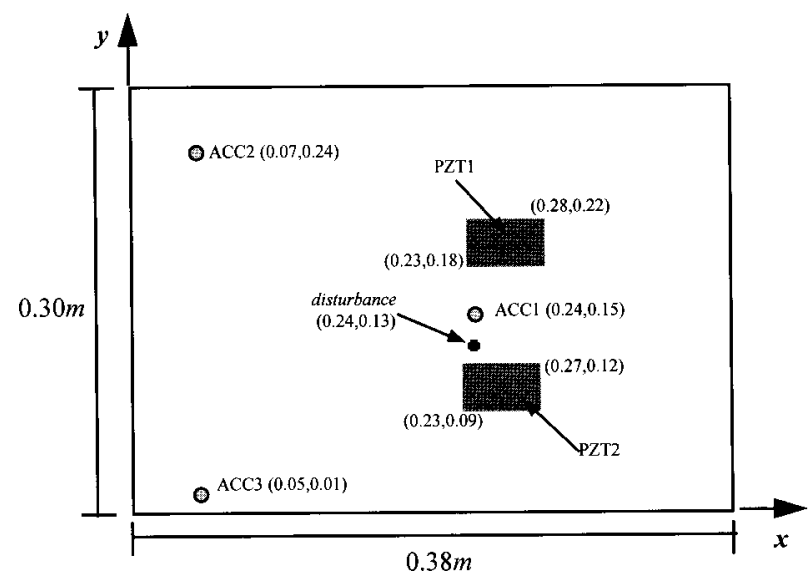

FIG. 6. Optimum actuator/sensor configuration (coordinates in meter)

expense of a significant violation in the control effort penalty. On the other hand, the control effort is reduced (i.e., the value of the constraint approaches the "ideal" value of 1.0) as the number of PZT patches is increased. This is because as the number of actuators is increased the match between the "ideal" and "actual" unit modal control forces is better and the control spillover to undesired modes is reduced.

From the 30 available solutions, the ninth configuration when using two PZTs is selected to be implemented in this example. In this configuration both actuators are driven inphase which yields a reduction of $9.9 \mathrm{~dB}$ in the total radiated power and a small violation in the control effort limit of 1.5.

b. Optimum sensor design. The mechanical model of accelerometer sensors is simply given as the value of the mode shape at the sensor location. The design variables in this optimization problem [i.e., the elements of $\{b\}$ in Eq. (20)] are the coordinates of the sensors $\left(x_{s j}, y_{s j}\right)$ for $j$ $=1, \ldots, N_{E}$. The physical domain optimization problem to design the sensors is again exactly in the same form as the case of the actuator design using the GA. Results are obtained for sensing configurations consisting of one, two, and three accelerometers (i.e., $N_{E}=1, \ldots, 3$ ). The performance of the best solutions of ten GA analyses is again evaluated in the same way as for the case of the actuators. Figure 5 shows the power reduction and control effort constraint for the 30 sensing configurations. To obtain these results, the "ideal" unit modal control forces found in the modal domain optimization are used (see Table I). The results in the third figure show that using only one sensor (i.e., minimizing the error at only one location) does not yield enough amplitude to the control input $U_{k}(\omega)$ to induce a significant attenuation in the total radiated power. The attenuation for this case is about 4 $\mathrm{dB}$. It can also be observed that the control effort constraint is not active [i.e., $\left.g_{1}^{c}\left(u_{n l}, \xi_{n l}\right)=0.5<1.0\right]$. As can be seen, as the number of sensors is increased there is an increase (in the average) in the reduction of the total radiated power. On the other hand, the modal control effort is very low with one sensor and also increases with the number of sensors. Based on these results the ninth configuration with three accelerometers is selected. This configuration (in conjunction with the "ideal" control forces) yields a power reduction of $11.0 \mathrm{~dB}$ and again a small control effort violation [i.e., $g_{1}^{c}\left(u_{n l}, \xi_{n l}\right)$ $=1.2>1.0]$.

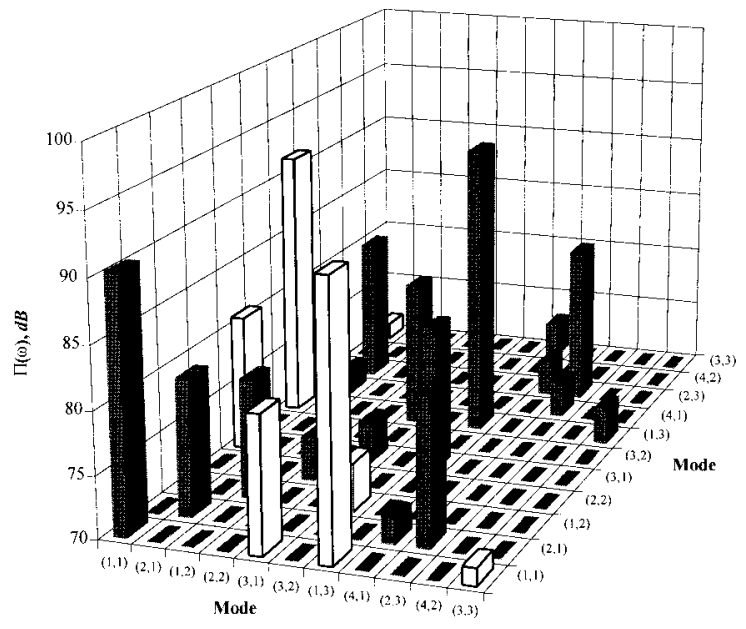

FIG. 7. Modal contributions to controlled power using the $1 \mathrm{I} 1 \mathrm{O}$ "actual" configuration. Total radiated power: $91.1 \mathrm{~dB}$.

\section{Optimum actuator/sensor configuration}

A schematic of the selected actuator/sensor configuration [i.e., 2-PZT(9) and 3-ACC(9) in Figs. 10 and 11] is shown in Fig. 6. The "actual" modal control and modal error sensor components are again presented in Table I. As can be seen, the "actual" unit modal control forces show a very good agreement to the "ideal" values. The most significant discrepancy between the "actual" and "ideal" values is the change in phase in the first component. On the other hand, when compared to the "ideal" values, the "actual" modal error parameters show some differences in terms of the relative observability of the modes and phases. From the results in the previous section, these differences do not seem to affect the performance of the control system. Once the actuators and sensors are designed, the performance of the complete "actual" system is then compared to the performance of the "ideal" one obtained in the modal domain optimization.

The magnitude of the control input using the selected "actual" configuration is $19.1 \mathrm{~V}$. This configuration yields a total reduction in the radiated power of $10.7 \mathrm{~dB}$ that is very close to the "ideal" attenuation predicted in the modal domain of $9.9 \mathrm{~dB}$. The modal contributions to the total radiated power when implementing the "actual" control system are shown in Fig. 7. This figure can be compared to the "ideal" reductions previously shown in Fig. 3. As can be seen, both results are very similar. Note that the selected actuators/ sensor configuration is only one of the 900 available combinations. In practice, the designer could check the performances of several configurations. Here, only one configuration was investigated to illustrate the method.

\section{CONCLUSIONS}

A new approach for the optimum design of actuators and error sensors to be implemented in ASAC applications has been developed. The formulation is based in a multi-level optimization procedure. The upper level is defined in the modal domain and solves for the optimum relative controllability and observability of the modes that the control system should implement in order to minimize the total radiated 
acoustic power. The optimum results at the upper level are used in the lower level or physical domain optimization problems in order to find the actuators and sensors to be implemented in the structure. The formulation allows the use of either numerical techniques or experimental results during the design process. Therefore, the proposed design approach can be readily applied for the design of control systems for complex structures with complex excitations. In this paper, the developed optimum design formulations were implemented for the case of a simply supported plate. The simply supported plate was studied because both its structural and acoustic responses are well understood which permitted to obtain a better understanding of results and the evaluation of the performance of the design method.

\section{ACKNOWLEDGMENTS}

The authors gratefully acknowledge the support of this work by the Office of Naval Research (Grant No. ONRN00014-92-J1170) and the State Council of Higher Education for Virginia (Commonwealth Graduate Fellowship).

${ }^{1}$ C. R. Fuller, "Apparatus and methods for global noise reduction," U.S. Patent No. 4,715,599 (1987).

${ }^{2}$ C. R. Fuller, S. J. Elliot, and P. A. Nelson, Active Control of Vibration (Academic, San Diego, CA, 1996).

${ }^{3}$ B. T. Wang, R. A. Burdisso, and C. R. Fuller, "Optimal placement of piezoelectric actuators for active control of sound radiation from elastic plates," Proceedings of Noise-Con 91, Tarrytown, New York, 14-16 July 1991, 267-275 (1991).

${ }^{4}$ C. E. Ruckman and C. R. Fuller, "Using multiple regression and subset selection to optimize actuator locations in feedforward active control systems", J. Acoust. Soc. Am. 92, 2410(A) (1992).

${ }^{5}$ R. H. Cabell, H. C. Lester, G. P. Mathur, and B. N. Tran, "Optimization of actuator arrays for aircraft interior noise control," 15th AIAA Aeroacoustics Conference, Long Beach, California, 25-27 October 1993, 1-10 (1993).

${ }^{6}$ R. A. Burdisso and C. R. Fuller, "Design of active structural acoustic control systems by eigenproperty assignment," J. Acoust. Soc. Am. 96, 1582-1591 (1994).

${ }^{7}$ M. T. Simpson and C. H. Hansen, "Use of genetic algorithms for optimizing vibration actuator placement for minimizing sound transmission into enclosed spaces," Proceedings of Smart Structures and Materials, San Diego, California, 26-29 February 1996.

${ }^{8}$ H. M. Rodriguez and R. A. Burdisso, "Sensitivity analysis for feedforward control system design," J. Acoust. Soc. Am. 98, 3352-3359 (1995).

${ }^{9}$ F. Fahy, Sound and Structural Vibration (Academic, Orlando, FL, 1985).

${ }^{10}$ M. C. Junger and D. Feit, Sound, Structures and Their Interaction (Acoustical Society of America, Woodbury, NY, 1993).

${ }^{11}$ R. D. Ciskowski and C. A. Brebbia, Boundary Element Methods in Acoustics (Comp. Mechanics Publications, Boston, 1991).

${ }^{12}$ H. M. Rodríguez, "Optimum design for feedforward structural-acoustic control of complex structural systems," Doctor of Philosophy Dissertation, Department of Mechanical Engineering, VPI\&SU (1996).

${ }^{13}$ The MathWorks, Inc., Optimization Toolbox User's Guide (Natick, MA, 1992).

${ }^{14}$ E. K. Dimitriadis, C. R. Fuller, and C. R. Rogers, "Piezoelectric actuators for distributed vibration excitation of thin plates," J. Vib. Acoust. 113, 100-107 (1989).

${ }^{15}$ D. E. Goldberg, Genetic Algorithms in Search, Optimization and Machine Learning (Addison-Wesley, Reading, MA, 1989). 